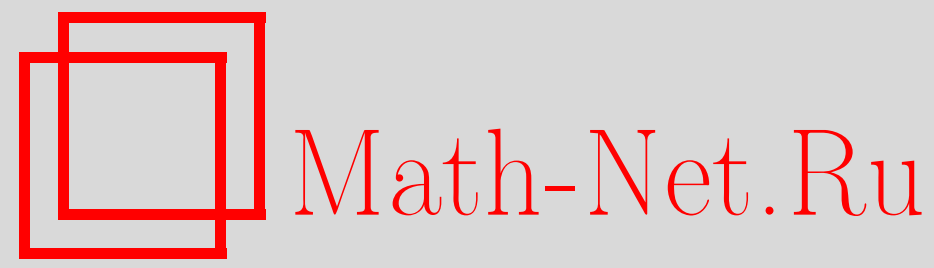

И. А. Круглов, Авторегрессия на конечной абелевой группе с марковской входной последовательностью, Матем. вопр. криптогр., 2011, том 2, выпуск 4, 25-36

DOI: https://doi.org/10.4213/mvk41

Использование Общероссийского математического портала Math-Net.Ru подразумевает, что вы прочитали и согласны с пользовательским соглашением

http://www . mathnet.ru/rus/agreement

Параметры загрузки:

IP : 54.84 .234 .179

26 апреля 2023 г., 04:20:58 
УДК: 519.217.2

\title{
Авторегрессия на конечной абелевой группе с марковской входной последовательностью
}

\author{
И. А. Круглов \\ ООО «Центр сертификационных исследований», Москва
}

Получено 23.VI.2011

В статье доказан аналог принципа сходимости Б.М.Клосса для последовательностей случайных величин из указанной в заглавии схемы суммирования случайных величин со значениями в конечных абелевых группах.

Ключевые слова: цепи Маркова на группах, условные распределения, предельные теоремы

Autoregression on a finite Abelian group controlled by a Markov chain

\section{A. Kruglov}

LLC Certification Research Center, Moscow

Abstract. An analog of convergence principle due to B.M. Kloss is proved for autoregressive sequences of random variables on a finite Abelian group controlled by a Markov chain.

Key words: Markov chains on groups, conditional distributions, limit theorems

Citation: Mathematical Aspects of Cryptography, 2011, vol. 2, no. 4, pp. 25-36 (Russian). 
В статье рассматривается следующий способ образования случайных величин, который получил название схемы авторегрессии на конечной группе.

Пусть

$$
\alpha_{1}, \alpha_{2}, \ldots
$$

- конечная простая однородная неразложимая цепь Маркова с множеством состояний $\{1,2, \ldots, n\}$, матрицей переходных вероятностей $P=\|p(i, j)\|_{i, j=1}^{n}$ и некоторым положительным вектором начального распределения, $(G ; \cdot)-$ конечная абелева группа. Предположим, что для каждых $i, j \in\{1,2, \ldots, n\}$ задана последовательность $\left\{\xi_{i, j}^{(N)}\right\}$ одинаково распределенных со случайной величиной $\xi_{i, j}$ случайных величин со значениями в группе $G$. Будем предполагать, что случайные величины $\xi_{i, j}^{(N)}, N \geq 1, i, j \in\{1, \ldots, n\}$, и цепь Маркова (1) независимы в совокупности. Предположим, что задан автоморфизм группы $G$ :

$$
\varphi: G \rightarrow G .
$$

Определим случайные величины со значениями в группе $G$ :

$$
\zeta^{(N)}=\left(\left(\ldots\left(\xi_{\alpha_{1}, \alpha_{2}}^{(1)}\right)^{\varphi} \cdot \ldots\right)^{\varphi} \cdot \xi_{\alpha_{N-1}, \alpha_{N}}^{(N-1)}\right)^{\varphi} \cdot \xi_{\alpha_{N}, \alpha_{N+1}}^{(N)}, \quad N \geq 1 .
$$

Для любого $N \geq 1$ пусть

$$
P^{N}=\left\|p^{(N)}(i, j)\right\|_{i, j=1}^{n}
$$

- матрица переходных вероятностей цепи Маркова $\left\{\alpha_{t}\right\}$ за $N$ шагов. Для таких $i, j \in\{1, \ldots, n\}, N \geq 1$, что $p^{(N)}(i, j)>0$, обозначим через $\zeta_{i, j}^{(N)}$ случайную величину со значениями в группе $G$, распределение которой совпадает с условным распределением $\zeta^{(N)}$ при условии $\alpha_{1}=i, \alpha_{N+1}=j$. Нас будет интересовать предельное поведение последовательностей распределений случайных величин $\zeta_{i, j}^{(N)}$ при $N \rightarrow \infty$.

Последовательность (2) в случае $n=1$, т. е. схема авторегрессии в случае, когда $\left\{\alpha_{t}\right\}$ - последовательность независимых одинаково распределенных случайных величин, исследовалась в [3]. Условия предельной равномерности распределений случайных величин $\zeta_{i, j}^{(N)}$ на группе $G$ при $N \rightarrow \infty$ указаны автором в [4].

Введем обозначения, необходимые для формулировки основной теоремы. Пусть ord $\varphi$ - порядок автоморфизма $\varphi, d-$ число циклических классов цепи Маркова (1), $f$ - наименьшее общее кратное чисел $d$ и ord $\varphi$. Рассмотрим ориентированный граф $Г$ (см. [1]) с множеством вершин $\{1,2, \ldots, n\}$ и множеством ориентированных ребер $\left\{e_{i, j}^{+1}, e_{j, i}^{-1} \mid i, j \in\{1, \ldots, n\}, p^{(f)}(i, j)>0\right\}$. 
Путем в Г назовем произвольную последовательность ориентированных ребер вида

$$
z=e_{i_{1}, i_{2}}^{\varepsilon_{1}}, e_{i_{2}, i_{3}}^{\varepsilon_{2}}, \ldots, e_{i_{k}, i_{k+1}}^{\varepsilon_{k}} .
$$

Путь $z$ вида (3) называется петлей, если $i_{1}=i_{k+1}$. Для любых $i, j \in$ $\in\{1, \ldots, n\}, R \in \mathbb{Z}$ обозначим через $L_{R}(i, j)$ множество всех путей $z$ вида (3), для которых $i_{1}=i, i_{k+1}=j, \varepsilon_{1}+\varepsilon_{2}+\ldots+\varepsilon_{k}=R$, при этом в случае, когда $L_{R}(i, j) \neq \varnothing$, для любого $z \in L_{R}(i, j)$ мы будем использовать обозначение $l(z)=R$.

Для произвольной случайной величины $\xi$ со значениями в $G$ обозначим через $\Omega(\xi)$ носитель распределения $\xi$. Для любых таких $i, j \in\{1, \ldots, n\}$, что $p^{(f)}(i, j)>0$, определим подмножества элементов группы $G$ :

$$
\bar{\Omega}\left(e_{i, j}^{+1}\right)=\Omega\left(\zeta_{i, j}^{(f)}\right), \quad \bar{\Omega}\left(e_{j, i}^{-1}\right)=\Omega\left(\zeta_{i, j}^{(f)}\right)^{-1} .
$$

Пути $z$ вида (3) поставим в соответствие множество элементов группы $G$ :

$$
\bar{\Omega}(z)=\bar{\Omega}\left(e_{i_{1}, i_{2}}^{\varepsilon_{1}}\right) \cdot \bar{\Omega}\left(e_{i_{2}, i_{3}}^{\varepsilon_{2}}\right) \cdot \ldots \cdot \bar{\Omega}\left(e_{i_{k}, i_{k+1}}^{\varepsilon_{k}}\right) .
$$

Пусть $D_{1}, D_{2}, \ldots, D_{d}$ - циклические классы состояний цепи Маркова (1). Можно считать, что для любого $\mu \in\{1, \ldots, d\}$ из состояний класса $D_{\mu}$ в цепи Маркова (1) возможен переход только в состояния класса $D_{\mu+1}$ (здесь $D_{d+1}=D_{1}$ ). Так как $d$ делит $f$, то (см. [6]) существует натуральное число $N_{0}$, при котором для любых $\mu \in\{1, \ldots, d\}, i, j \in D_{\mu}, N \geq N_{0}$

$$
p^{(N \cdot f)}(i, j)>0 .
$$

Отсюда следует, что для любых $\mu \in\{1, \ldots, d\}, i, j \in D_{\mu}, R \in \mathbb{Z}$ множество $L_{R}(i, j) \neq \varnothing$.

Зафиксируем произвольно петлю $z_{1} \in L_{1}(1,1)$ и элемент $x_{1} \in \bar{\Omega}\left(z_{1}\right)$; пусть $Z^{(1)}-$ множество всех путей вида (3), удовлетворяющих соотношению $i_{1}=i_{k+1}=1$; обозначим через $H_{1}$ минимальную подгруппу среди всех подгрупп $L$ группы $G$, для которых при любом $z \in Z^{(1)}$ выполнено включение

$$
\bar{\Omega}(z) \subset x_{1}^{l(z)} \cdot L .
$$

Для произвольных $i, j \in\{1, \ldots, n\}$ пусть $\nu, \mu \in\{1, \ldots, d\}$ таковы, что $i \in$ $\in D_{\nu}, j \in D_{\mu}$; положим

$$
\nu(i, j)= \begin{cases}\mu-\nu, & \text { если } \mu \geq \nu, \\ \mu-\nu+d, & \text { если } \mu<\nu,\end{cases}
$$

тогда $\nu(i, j) \in\{0,1, \ldots, d-1\}$. 
Из соотношения (5) следует, что для любых $N \geq 0, i, j \in\{1, \ldots, n\}$ определены случайные величины $\zeta_{i, j}^{\left(\nu(i, j)+N \cdot d+N_{0} \cdot f\right)}$.

Для любых $i, j \in\{1, \ldots, n\}, t \in\{0,1, \ldots, f / d-1\}$ зафиксируем произвольно элементы

$$
\tau_{i, j}^{(t)} \in \Omega\left(\zeta_{i, j}^{\left(\nu(i, j)+t \cdot d+N_{0} \cdot f\right)}\right)
$$

и для любых $i, j \in\{1, \ldots, n\}, N \geq 0$, таких что $p^{(\nu(i, j)+N \cdot d)}(i, j)>0$, определим элементы $\sigma_{i, j}^{(N)}$ группы $G$ следующим образом.

Каждое число $N \geq 0$ может быть однозначно представлено в виде

$$
N=s_{N}+\frac{f}{d} \cdot k_{N}
$$

где $k_{N} \geq 0, s_{N} \in\{0,1, \ldots, f / d-1\}$, если $\nu(i, j)>0$ и $s_{N} \in\{1,2, \ldots, f / d\}$, если $\nu(i, j)=0$. Пусть

$$
t_{N}= \begin{cases}f / d-\left(s_{N}+1\right), & \text { если } \nu(i, j)>0 \\ f / d-s_{N}, & \text { если } \nu(i, j)=0\end{cases}
$$

тогда $t_{N} \in\{0,1, \ldots, f / d-1\}$.

Далее, для любого $\mu \in\{1, \ldots, d\}$ через $\bar{\mu} \in\{0,1, \ldots, m-1\}$ будем обозначать наименьший неотрицательный вычет $\mu$ по модулю $m=$ НОД $(d, m)$. Положим

$$
\sigma_{i, j}^{(N)}=\left(\left(x_{1}\right)^{\varphi^{\bar{\mu}}}\right)^{-N_{0}-k_{N}-1} \cdot\left(\tau_{j, i}^{\left(t_{N}\right)}\right)^{\varphi^{\left(\nu(i, j)+s_{N} \cdot d\right)}} .
$$

Следующая теорема является аналогом принципа сходимости Б. М. Клосса (см. [5]) для последовательностей случайных величин $\zeta_{i, j}^{(N)}$.

Теорема 1. Для любых $i \in\{1, \ldots, n\}, \mu \in\{1, \ldots, d\}, j \in D_{\mu}$ последовательность распределений случайных величин

$$
\sigma_{i, j}^{(N)} \cdot \zeta_{i, j}^{(N)}, N \geq 1, p^{(\nu(i, j)+N \cdot d)}(i, j)>0,
$$

имеет своим пределом при $N \rightarrow \infty$ равномерное распределение на подгруппе $\left(H_{1}\right)^{\varphi^{\bar{\mu}}}$.

Доказательство. Рассмотрим цепь Маркова

$$
\alpha_{1}, \alpha_{f+1}, \ldots, \alpha_{f \cdot N+1}, \ldots
$$


с множеством состояний $\{1, \ldots, n\}$, матрицей переходных вероятностей, равной матрице $P^{f}$, и начальным распределением, совпадающим с начальным распределением цепи Маркова (1). Множества $D_{1}, D_{2}, \ldots, D_{d}-$ классы существенных состояний цепи Маркова (8).

Произвольной паре состояний $i, j \in\{1, \ldots, n\}$, для которых $p^{(f)}(i, j)>0$, поставим в соответствие последовательность $\left\{\widehat{\xi}_{i, j}^{(N)}\right\}$ одинаково распределенных со случайной величиной $\zeta_{i, j}^{(f)}$ случайных величин со значениями в группе $G$. Будем предполагать, что случайные величины $\widehat{\xi}_{i, j}^{(N)}, N \geq 1, i, j \in\{1, \ldots, n\}$, и цепь Маркова (1) независимы в совокупности.

Определим следующие случайные величины со значениями в группе $G$ (см. §3 работы [2]):

$$
\eta^{(N)}=\widehat{\xi}_{\alpha_{1}, \alpha_{f+1}}^{(1)} \cdot \widehat{\xi}_{\alpha_{f+1}, \alpha_{2 f+1}}^{(2)} \ldots \cdot \widehat{\xi}_{\alpha_{f}(N-1)+1}^{(N-1), \alpha_{f N+1}}, \quad N \geqslant 1 .
$$

Для любых $N \geq 1, i, j \in\{1, \ldots, n\}$, удовлетворяющих условию $p^{(f \cdot N)}(i, j)>0$, рассмотрим случайную величину $\eta_{i, j}^{(N)}$ со значениями в группе $G$, распределение которой совпадает с условным распределением случайной величины $\eta^{(N)}$ при условии $\alpha_{1}=i, \alpha_{f N+1}=j$, и докажем, что распределения случайных величин $\zeta_{i, j}^{(N \cdot f)}$ и $\eta_{i, j}^{(N)}$ совпадают.

Так как $\operatorname{ord} \varphi$ делит $f$, то из (2) следует равенство

$$
\begin{gathered}
\zeta^{(N \cdot f)}=\left(\ldots\left(\xi_{\alpha_{1}, \alpha_{2}}^{(1)}\right)^{\varphi} \cdot \ldots\right)^{\varphi} \cdot \xi_{\alpha_{f}, \alpha_{f+1}}^{(f)} \times \\
\times\left(\ldots\left(\xi_{\alpha_{f+1}, \alpha_{f+2}}^{(f+1)}\right)^{\varphi} \cdot \ldots\right)^{\varphi} \cdot \xi_{\alpha_{2 \cdot f}, \alpha_{2 \cdot f+1}}^{(2 \cdot f)} \ldots \times \\
\times\left(\ldots\left(\xi_{\alpha_{(N-1) \cdot f+1}((N-1) \cdot f(N-1) \cdot f+2}^{(N+1)}\right)^{\varphi} \cdot \ldots\right)^{\varphi} \cdot \xi_{\alpha_{N \cdot f}, \alpha_{N \cdot f+1}}^{(N \cdot f)} .
\end{gathered}
$$

Из этого соотношения следует, что для любой последовательности $j_{1}, j_{2}, \ldots, j_{N+1} \in\{1, \ldots, n\}$, для которой

$$
p^{(f)}\left(j_{1}, j_{2}\right) \cdot p^{(f)}\left(j_{2}, j_{3}\right) \cdot \ldots \cdot p^{(f)}\left(j_{N}, j_{N+1}\right)>0,
$$

условное распределение $\zeta^{(N \cdot f)}$ при условии

$$
\alpha_{1}=j_{1}, \alpha_{f+1}=j_{2}, \ldots, \alpha_{N \cdot f+1}=j_{N+1}
$$

совпадает с распределением произведения независимых случайных величин,

$$
\psi_{1} \cdot \psi_{2} \cdot \ldots \cdot \psi_{N},
$$


в котором для каждого $k \in\{1, \ldots, N\}$ распределение $\psi_{k}$ совпадает с распределением $\zeta_{j_{k}, j_{k+1}}^{(f)}$. Однако распределение произведения (10) совпадает с распределением случайной величины $\eta^{(N)}$ при условии реализации цепочки состояний $j_{1}, j_{2}, \ldots, j_{N+1}$ в цепи Маркова (8) в первых $N+1$ испытаниях; вероятность этого события равна вероятности осуществления события (9) для цепи Маркова (1). Из формулы полной вероятности следует совпадение распределений $\zeta_{i, j}^{(N \cdot f)}$ и $\eta_{i, j}^{(N)}$.

В [2] описано предельное поведение распределений случайных величин $\eta_{i, j}^{(N)}$, при этом введена некоторая подгруппа группы $G$, которая совпадает с определенной выше подгруппой $H_{1}$.

Аналогично случаю $j=1$ для каждого $j \in\{2, \ldots, n\}$ зафиксируем произвольно петлю $z_{j} \in L_{1}(j, j)$ и элемент $x_{j} \in \bar{\Omega}\left(z_{j}\right)$; пусть $Z^{(j)}$ - множество всех путей вида (3), удовлетворяющих соотношению $i_{1}=i_{k+1}=j$; обозначим через $H_{j}$ минимальную подгруппу среди всех подгрупп $L$ группы $G$, для которых выполнены включения

$$
\bar{\Omega}(z) \subset x_{j}^{l(z)} \cdot L, z \in Z^{(j)} .
$$

Зафиксируем $i \in\{1, \ldots, n\}, \mu \in\{1, \ldots, d\}, j \in D_{\mu}$, введем элементы

$$
y_{i, j}^{(N)}=x_{j}^{-N_{0}-k_{N}-1} \cdot\left(\tau_{j, i}^{\left(t_{N}\right)}\right)^{\varphi^{\left(\nu(i, j)+s_{N} \cdot d\right)}}, \quad N \geq 1,
$$

группы $G$ и докажем, что последовательность распределений случайных величин

$$
y_{i, j}^{(N)} \cdot \zeta_{i, j}^{(N)}, N \geq 1, p^{(\nu(i, j)+N \cdot d)}(i, j)>0,
$$

имеет своим пределом при $N \rightarrow \infty$ равномерное распределение на подгруппе $H_{j}$.

Для любых $u \in\{1, \ldots, n\}, N \geq 1$, удовлетворяющих условию $p^{(N \cdot f)}(u, j)>0$, зафиксируем произвольно элемент

$$
g_{u, j}^{(N)} \in \Omega\left(\zeta_{u, j}^{(N \cdot f)}\right) .
$$

Отметим, что $u \in D_{\mu}$ и что $\nu(i, j)=\nu(i, u)$. Из теоремы $\S 3$ в [2] следует, что

$$
\begin{gathered}
\mathbf{P}\left(\left(g_{u, j}^{(N)}\right)^{-1} \cdot \zeta_{u, j}^{(N \cdot f)}=g\right)=0, \quad g \in G \backslash H_{j}, N \geq 0, p^{(N \cdot f)}(u, j)>0, \\
\mathbf{P}\left(\left(g_{u, j}^{(N)}\right)^{-1} \cdot \zeta_{u, j}^{(N \cdot f)}=h\right)=\frac{1}{\left|H_{j}\right|} \cdot(1+o(1)), \quad h \in H_{j}, N \rightarrow \infty .
\end{gathered}
$$


По формуле полной вероятности с учетом (13) для любого такого $N \geq 0$, что $p^{(\nu(i, j)+N \cdot d)}(i, j)>0$, и любого $g \in G$

$$
\begin{gathered}
\mathbf{P}\left(y_{i, j}^{(N)} \cdot \zeta_{i, j}^{(\nu(i, j)+N \cdot d)}=g\right)=p^{(\nu(i, j)+N \cdot d)}(i, j)^{-1} \times \\
\times \sum_{u \in D_{\mu}} p^{\left(\nu(i, u)+s_{N} \cdot d\right)}(i, u) \cdot p^{\left(k_{N} \cdot f\right)}(u, j) \times \\
\times\left[\sum_{h \in H_{j}} \mathbf{P}\left(y_{i, j}^{(N)} \cdot \zeta_{i, u}^{\left(\nu(i, u)+s_{N} \cdot d\right)} \cdot g_{u, j}^{\left(k_{N}\right)}=g \cdot h^{-1}\right) \times\right. \\
\left.\times \mathbf{P}\left(\left(g_{u, j}^{\left(k_{N}\right)}\right)^{-1} \cdot \zeta_{u, j}^{\left(k_{N} \cdot f\right)}=h\right)\right] .
\end{gathered}
$$

Для любых таких $u \in D_{\mu}, N \geq 0$, что $p^{\left(\nu(i, u)+s_{N} \cdot d\right)}(i, u)>0$, рассмотрим произвольный элемент

$$
\rho \in \Omega\left(\zeta_{i, u}^{\left(\nu(i, u)+s_{N} \cdot d\right)}\right)
$$

Справедливо включение

$$
\begin{gathered}
\left(\tau_{j, i}^{\left(t_{N}\right)}\right)^{\varphi^{\left(\nu(i, j)+s_{N} \cdot d\right)}} \cdot \rho \cdot g_{u, j}^{\left(k_{N} \cdot f\right)} \in \Omega\left(\zeta_{j, i}^{\left(\nu(j, i)+t_{N} \cdot d+N_{0} \cdot f\right)}\right)^{\varphi^{\left(\nu(i, j)+s_{N} \cdot d\right)}} \times \\
\times \Omega\left(\zeta_{i, u}^{\left(\nu(i, j)+s_{N} \cdot d\right)}\right) \cdot \Omega\left(\zeta_{u, j}^{\left(k_{N} \cdot f\right)}\right) \subset \bar{\Omega}(z),
\end{gathered}
$$

где $z \in L_{R}(j, j)$,

$$
\begin{gathered}
\quad R=\frac{1}{f} \cdot\left(\nu(j, i)+t_{N} \cdot d+N_{0} \cdot f+\nu(i, j)+s_{N} \cdot d+k_{N} \cdot f\right)= \\
=N_{0}+k_{N}+\frac{1}{f} \cdot\left(\nu(j, i)+\nu(i, j)+\left(s_{N}+t_{N}\right) \cdot d\right)=N_{0}+k_{N}+1 .
\end{gathered}
$$

Из условия (11), выполненного при $L=H_{j}$, следует, что

$$
\bar{\Omega}(z) \subset x_{j}^{N_{0}+k_{N}+1} \cdot H_{j} .
$$

Таким образом, из соотношений (6), (16), (17) для любых таких $u \in D_{\mu}, N \geq 0$, что $p^{\left(\nu(i, u)+s_{N} \cdot d\right)}(i, u)>0$, следует включение

$$
\Omega\left(y_{i, j}^{(N)} \cdot \zeta_{i, u}^{\left(\nu(i, u)+s_{N} \cdot d\right)} \cdot g_{u, j}^{\left(k_{N}\right)}\right) \subset H_{j}
$$

Из соотношений (18) и (15) следует, что для любого $g \in G \backslash H_{j}$ и любого $N \geq 0$, удовлетворяющего условию $p^{(\nu(i, j)+N \cdot d)}(i, j)>0$, верно равенство

$$
\mathbf{P}\left(y_{i, j}^{(N)} \cdot \zeta_{i, j}^{(\nu(i, j)+N \cdot d)}=g\right)=0 .
$$


Из теории конечных цепей Маркова ([6]) следует, что существуют числа $p(u)>0, u \in D_{\mu}$, для которых

$$
p^{(\nu(i, u)+N \cdot d)}(i, u)=p(u) \cdot(1+o(1)), \quad N \rightarrow \infty ;
$$

а также

$$
\sum_{u \in D_{\mu}} p(u)=1
$$

Наконец, согласно (18), для любых $u \in D_{\mu}, N \geq 0$, удовлетворяющих условию $p^{\left(\nu(i, u)+s_{N} \cdot d\right)}(i, u)>0$, верно равенство

$$
\sum_{h \in H_{j}} \boldsymbol{P}\left(y_{i, j}^{(N)} \cdot \zeta_{i, u}^{\left(\nu(i, u)+s_{N} \cdot d\right)} \cdot g_{u, j}^{\left(k_{N}\right)}=g \cdot h^{-1}\right)=1, g \in H_{j} .
$$

Подставив (14), (20) в (15) и используя (21), (22), получим для любого $g \in H_{j}$

$$
\boldsymbol{P}\left(y_{i, j}^{(N)} \cdot \zeta_{i, j}^{(\nu(i, j)+N \cdot d)}=g\right)=\frac{1}{\left|H_{j}\right|} \cdot(1+o(1)), \quad N \rightarrow \infty .
$$

Из (19) и (23) вытекает доказываемое утверждение о последовательности (12).

Исследуем теперь связи между подгруппами $H_{j}$ для различных $j \in$ $\in\{1, \ldots, n\}$. Докажем, что для любых $i, j \in\{1, \ldots, n\}$ верны равенства

$$
\begin{aligned}
H_{j} & =\left(H_{i}\right)^{\varphi^{\nu(i, j)}}, \\
x_{j} \cdot H_{j} & =\left(x_{i} \cdot H_{i}\right)^{\varphi^{\nu(i, j)}} .
\end{aligned}
$$

Зафиксируем произвольные $i, j \in\{1, \ldots, n\}$. Из соотношений (19) и (23) следует, что существует натуральное число $N$, для которого порядок группы $G$ делит $N$, и

$$
\begin{gathered}
\Omega\left(\zeta_{j, j}^{(f \cdot N)}\right)=H_{j}, \\
\Omega\left(\zeta_{j, j}^{(f \cdot(N+1))}\right)=x_{j} \cdot H_{j} .
\end{gathered}
$$

Выберем произвольный элемент

$$
\rho \in \Omega\left(\zeta_{j, i}^{\left(f-\nu(i, j)+N_{0} \cdot f\right)}\right)
$$

пусть

$$
\tau=\left(x_{i}^{1+2 \cdot N_{0}} \cdot \rho^{-1}\right)^{\varphi^{\nu(i, j)}} .
$$


Из соотношений (2), (28) следует, что

$$
\left[\tau_{i, j}^{(0)} \cdot \Omega\left(\zeta_{j, j}^{(f \cdot N)}\right)\right]^{\varphi^{(f-\nu(i, j))}} \cdot \rho \subset \Omega\left(\zeta_{i, i}^{\left(f \cdot\left(1+2 \cdot N_{0}+N\right)\right)}\right) .
$$

Из (11) следует, что

$$
\Omega\left(\zeta_{i, i}^{\left(f \cdot\left(1+2 \cdot N_{0}+N\right)\right)}\right) \subset x_{i}^{1+2 \cdot N_{0}+N} \cdot H_{i} .
$$

Заметим, что $x_{i} \cdot H_{i}=H_{i} \cdot x_{i}$. Так как порядок элемента $x_{i}$ делит $N$, то из (26), (29), (30) и (31) следует включение

$$
H_{j} \subset\left(\tau_{i, j}^{(0)}\right)^{-1} \cdot H_{i}^{\varphi^{\nu(i, j)}} \cdot \tau .
$$

Согласно (32), $\left|H_{j}\right| \leq\left|H_{i}\right|$. Проводя аналогичные рассуждения, можно показать, что $\left|H_{i}\right| \leq\left|H_{j}\right|$, т. е. $\left|H_{j}\right|=\left|H_{i}\right|$.

Но тогда из (32) и условия коммутативности группы $G$ следует равенство

$$
H_{j}=\left(\tau_{i, j}^{(0)}\right)^{-1} \cdot \tau \cdot H_{i}^{\varphi^{\nu(i, j)}}
$$

Из (33) следует, что

$$
\left(\tau_{i, j}^{(0)}\right)^{-1} \cdot \tau \in H_{j}
$$

откуда вытекает доказываемое равенство (24).

Для доказательства (25) воспользуемся (27) и, проводя аналогичные (28)-(33) рассуждения, получим равенство

$$
x_{j} \cdot H_{j}=\left(\tau_{i, j}^{(0)}\right)^{-1} \cdot \tau \cdot x_{i}^{\varphi^{\nu(i, j)}} \cdot H_{i}^{\varphi^{\nu(i, j)}} .
$$

Теперь (25) следует из (34), (35) и (24).

Из соотношений (24), (25) следует, что для любых $\mu \in\{1, \ldots, d\}, j \in$ $\in D_{\mu}$ справедливы соотношения

$$
\begin{aligned}
H_{j} & =\left(H_{1}\right)^{\varphi^{\mu-1}}, \\
x_{j} \cdot H_{j} & =\left(x_{1} \cdot H_{1}\right)^{\varphi^{\mu-1}} .
\end{aligned}
$$

Докажем равенства

$$
\begin{aligned}
H_{1} & =\left(H_{1}\right)^{\varphi^{m}}, \\
x_{1} \cdot H_{1} & =\left(x_{1} \cdot H_{1}\right)^{\varphi^{m}} .
\end{aligned}
$$


Соотношение (38) следует из (39), докажем соотношение (39). Аналогично (27), существует натуральное число $k$, для которого верно равенство

$$
\Omega\left(\zeta_{1,1}^{(k \cdot f)}\right)=x_{1} \cdot H_{1}
$$

Множество $\Omega\left(\zeta_{1,1}^{(k \cdot f)}\right)$ есть объединение множеств $\bar{\Omega}(z)$, где петли $z \in L_{k}(1,1)$ имеют вид (3) при $\varepsilon_{1}=\varepsilon_{2}=\ldots=\varepsilon_{k}=+1$. Зафиксируем произвольно такую петлю $z$ и элемент $g \in \bar{\Omega}(z)$. Тогда из (4) и (2) следует равенство

$$
g=g_{1}^{\varphi^{k \cdot f-1}} \cdot g_{2}^{\varphi^{k \cdot f-2}} \cdot \ldots \cdot g_{k \cdot f-1}^{\varphi} \cdot g_{k \cdot f}
$$

для некоторых $g_{s} \in \Omega\left(\xi_{i_{s}, i_{s+1}}\right)$ и $i_{s} \in\{1, \ldots, n\}$, удовлетворяющих условиям $p\left(i_{s}, i_{s+1}\right)>0$ при всех $s \in\{1, \ldots, k \cdot f\}$, здесь $i_{1}=i_{k \cdot f+1}=1$.

Используя коммутативность группы $G$ и то, что числа $d, \operatorname{ord} \varphi$ делят $f$, равенство (41) можно переписать следующим образом:

$$
\begin{gathered}
g=\left[g_{k \cdot f-d+1}^{\varphi^{k \cdot f-1}} \cdot g_{k \cdot f-d+2}^{\varphi^{k \cdot f-2}} \cdot \ldots \cdot g_{k \cdot f}^{\varphi^{k \cdot f-d}} \cdot g_{1}^{\varphi^{k \cdot f-d-1}} \times\right. \\
\left.\times g_{2}^{\varphi^{k \cdot f-d-2}} \cdot g_{k \cdot f-d-1}^{\varphi} \cdot g_{k \cdot f-d}\right]^{\varphi^{d}} .
\end{gathered}
$$

Элемент в квадратных скобках в правой части (42) лежит в множестве

$$
\Omega\left(\zeta_{i_{d+1}, i_{d+1}}^{(k \cdot f)}\right) \subset x_{i_{d+1}} \cdot H_{i_{d+1}} .
$$

Согласно (37)

$$
x_{i_{d+1}} \cdot H_{i_{d+1}}=x_{1} \cdot H_{1} .
$$

Ввиду (40) и произвольности выбора $z, g$ из этих рассуждений следует включение

$$
\left(x_{1} \cdot H_{1}\right)^{\varphi^{d}} \subset x_{1} \cdot H_{1} .
$$

Однако отображение $\varphi^{d}$ биективно, поэтому согласно (43)

$$
\left(x_{1} \cdot H_{1}\right)^{\varphi^{d}}=x_{1} \cdot H_{1},
$$

откуда следует (39).

Из соотношений (36)-(39) следует, что для любых $\mu \in\{1, \ldots, d\}, j \in$ $\in D_{\mu}$ справедливы соотношения

$$
\begin{aligned}
H_{j} & =\left(H_{1}\right)^{\varphi^{\bar{\mu}}}, \\
x_{j} \cdot H_{j} & =\left(x_{1} \cdot H_{1}\right)^{\varphi^{\bar{\mu}}} .
\end{aligned}
$$


Из (44) и (45) для любых $i \in\{1, \ldots, n\}, \mu \in\{1, \ldots, d\}, j \in D_{\mu}$ следует равенство

$$
y_{i, j}^{(N)} \cdot H_{j}=\sigma_{i, j}^{(N)} \cdot H_{j} .
$$

Из (46) и доказанной выше сходимости распределений случайных величин последовательностей (12) к равномерному распределению на подгруппе $H_{j}$ следует, что для любых $i, j \in\{1, \ldots, n\}$ последовательность распределений случайных величин

$$
\sigma_{i, j}^{(N)} \cdot \zeta_{i, j}^{(N)}, \quad N \geq 1, p^{(\nu(i, j)+N \cdot d)}(i, j)>0,
$$

имеет своим пределом при $N \rightarrow \infty$ равномерное распределение на подгруппе $H_{j}$. С учетом (44) отсюда следует утверждение теоремы 1.

В случае, когда цепь Маркова (1) положительно регулярна, т. е. $d=1$, утверждение теоремы значительно упрощается. В этом случае число $f$ есть порядок автоморфизма $\varphi$, для любого $N \geq 1$

$$
\sigma_{i, j}^{(N)}=\left(x_{1}\right)^{-N_{0}-k_{N}-1} \cdot\left(\tau_{j, i}^{\left(t_{N}\right)}\right)^{\varphi^{s_{N}}}, \quad \tau_{i, j}^{\left(t_{N}\right)} \in \Omega\left(\zeta_{i, j}^{\left(t_{N}+N_{0} \cdot f\right)}\right),
$$

числа $k_{N}, s_{N}, t_{N}$ определяются соотношениями

$$
N=s_{N}+f \cdot k_{N}, \quad k_{N} \geq 0, \quad s_{N} \in\{1,2, \ldots, f\}, \quad t_{N}=f-s_{N} .
$$

Подгруппа $H_{1}$ и смежный класс $x_{1} \cdot H_{1}$ инвариантны относительно действия автоморфизма $\varphi$.

Следствие 1. Если иепь Маркова (1) положительно регулярна, то для любых $i, j \in\{1, \ldots, n\}$ последовательность распределений случайных величин

$$
\sigma_{i, j}^{(N)} \cdot \zeta_{i, j}^{(N)}, \quad N \geq 1, \quad p^{(N)}(i, j)>0,
$$

имеет своим пределом при $N \rightarrow \infty$ равномерное распределение на подгруппе $H_{1}$.

Следствие 2. Если иепь Маркова (1) положительно регулярна, тогда для любых $i, j \in\{1, \ldots, n\}$ последовательность распределений случайных величин

$$
\zeta_{i, j}^{(N)}, \quad N \geq 1, \quad p^{(N)}(i, j)>0,
$$

имеет своим пределом при $N \rightarrow \infty$ равномерное распределение на группе $G$ тогда и только тогда, когда группа $G$ не содержит собственной подгрупnы L, инвариантной относительно автоморфизма $\varphi$ и удовлетворяющей соотношению

$$
\bar{\Omega}(z) \subset x_{1}^{l(z)} \cdot L, \quad z \in Z^{(1)} .
$$

Утверждение следствия 2 в близкой формулировке было ранее доказано С. В. Глуховым. 


\section{Список литературы}

1. Бахтурин Ю. А. Основные структуры современной алгебры. - М.: Наука, Гл. ред. физ.-мат. лит., 1990. с. 320.

2. Горчинский Ю.Н., Круглов И.А., Капитонов В.М. Вопросы теории распределений на конечных группах. - В сб.: Труды по дискретной математике. Т. 1. - М.: ТВП, 1997, с. 85-112.

3. Егоров Б.А., Максимов Ю.И. Об одной последовательности случайных величин, принимающих значения из компактной коммутативной группы. - Теория вероятн. и ее примен., 1968, т. 13, вып. 4, с. 621-630.

4. Круглов И.А. Условия предельной равновероятности состояний регистров сдвига. - Математические вопросы криптографии, 2010, т. 1, вып. 2, с. 19-30.

5. Клосс Б. М. О вероятностных распределениях на бикомпактных топологических группах - Теория вероятн. и ее примен., 1959, т. 4, вып. 3, c. 255-290.

6. Романовский В. И. Дискретные цепи Маркова. - М.: Гостехиздат, 1949. 